\title{
Circulação extracorpórea por membrana (ECMO) em recém-nascido com insuficiência respiratória por síndrome de aspiração meconial: efeitos da administração de surfactante exógeno
}

\author{
Extracorporeal membrane oxygenation (ECMO) in a neonate with respiratory distress \\ due to meconium aspiration syndrome: Effect of the administration of exogenous surfactant
}

\author{
João Gilberto Maksoud-Filho' ${ }^{1}$, Edna Maria Albuquerque Diniz ${ }^{2}$, Maria Esther J. Ceccon ${ }^{3}$, \\ Ana Lúcia S. Galvani ${ }^{4}$, Maria D’Andrea A.B. Chamelian ${ }^{5}$, Maria Lúcia de Pinho ${ }^{6}$, Flávio Adolfo C. Vaz ${ }^{7}$
}

\section{Resumo}

Objetivos: apresentar a evolução clínica de recém-nascido portador de insuficiência respiratória grave neonatal secundária à sindrome de aspiração meconial tratado por circulação extracorpórea por membrana, ou, conforme o termo consagrado em língua inglesa, ECMO (Extracorporeal Membrane Oxygenation), o efeito do uso de surfactante exógeno neste caso e os custos do procedimento.

Método: descrição de um caso de síndrome de aspiração meconial, tratado na UCINE (Unidade de Cuidados Intensivos Neonatais) do Instituto da Criança Prof. Pedro de Alcantara, Hospital das Clínicas da Universidade de São Paulo.

Resultados: o suporte extracorpóreo teve a duração de 5 dias, sem complicações clínicas ou mecânicas. Surfactante exógeno de origem porcina foi administrado no $4^{\circ}$ dia, após o quê observamos uma melhora significativa na complacência pulmonar. O recém-nascido pôde então ser rapidamente decanulado. Os custos do tratamento foram compatíveis com a realidade nacional em relação a um recém-nascido criticamente enfermo.

Conclusões: a ECMO é indicada em casos de insuficiência respiratória neonatal que não respondam a outros tratamentos existentes. Deve ser disponível em Unidades de Tratamento Intensivo (UTIs) neonatais de hospitais terciários e ser empregada conforme critérios bem estabelecidos. A utilização de surfactante exógeno aparentemente antecipou a retirada da ECMO e, portanto, deve ser considerada em casos semelhantes. Os custos do tratamento justificam a organização de Equipes de ECMO nessas UTIs.

J Pediatr (Rio J) 2001; 77 (3): 243-8: aspiração de mecônio, insuficiência respiratória, recém-nascido, surfactantes pulmonares, circulação extracorpórea.

\begin{abstract}
Objectives: to present the clinical outcome of a newborn with severe respiratory distress secondary to meconium aspiration syndrome and treated by extracorporeal membrane oxygenation (ECMO); and to present the effect of the use of exogenous surfactant in this case and the cost of the procedure.

Methods: case report of a newborn with meconium aspiration syndrome and treated at the neonatal ICU of the Instituto da Criança Prof. Pedro de Alcantara, Hospital das Clínicas of the Universidade de São Paulo.

Results: ECMO was carried out for 5 days with no clinical or mechanical complications. On the $4^{\text {th }}$ day of ECMO, we administered porcine exogenous surfactant; a significant improvement in lung compliance was observed and the newborn was decannulated shortly after that. Treatment costs were compatible with the situation of healthcare in Brazil for treatment of critically ill newborn patients.

Conclusions: ECMO is indicated in cases of neonatal respiratory distress not responding to other treatments. The technique should be made available in neonatal Intensive Care Units (ICUs) of tertiary hospitals according to well-established protocols. The use of exogenous surfactant apparently allowed for earlier decannulation of the patient and should be considered in similar cases. The treatment costs do justify the organizing of ECMO teams in this type of ICUs.
\end{abstract}

J Pediatr (Rio J) 2001; 77 (3): 243-8: meconium aspiration, respiratory failure, infant, newborn, pulmonary surfactants, extracorporeal circulation,

1. Professor Doutor do Depto. de Pediatria da Faculdade de Medicina da Universidade de São Paulo. Assistente da Disciplina de Cirurgia Pediátrica do Instituto da Criança, Faculdade de Medicina da USP.

2. Professor Associado, Depto. de Pediatria, Disciplina de Neonatologia, Faculdade de Medicina da Univ. de São Paulo. Chefe da Unidade de Cuidados Intensivos Neonatais (UCINE) do Instituto da Criança "Professor Pedro de Alcantara", Faculdade de Medicina da USP.

3. Professor Doutor do Depto. de Pediatria da Fac. de Medicina da USP. Médico Assitente da Unidade de Cuidados Intensivos Neonatais (UCINE) do Instituto da Criança "Professor Pedro de Alcantara", Fac. de Medicina da USP.

4. Mestre em Pediatria pela Fac. de Medicina da USP. Médico Assistente da Unidade de Cuidados Intensivos Neonatais (UCINE) do Instituto da Criança "Professor Pedro de Alcantara", Fac. de Medicina da USP.

5. Pós-graduanda do Depto. de Pediatria da Fac. de Medicina da USP.

6. Pós-graduanda Depto. de Cirurgia da Faculdade de Medicina da USP.

7. Professor Titular do Depto. de Pediatria da Fac. de Medicina da USP. 
ECMO (Extracorporeal membrane oxygenation) ou ECLS (Extracorporeal Life Support) são termos empregados para descrever métodos de suporte extracorpóreo prolongado utilizados especialmente em recém nascidos portadores de insuficiência respiratória aguda que não respondem a formas "convencionais" de tratamento ${ }^{1}$. Mais de cem centros pediátricos no mundo dispõem rotineiramente dessa tecnologia, tendo tratado mais de 19.000 crianças até o momento. Apresentamos o primeiro caso de recém-nascido tratado com ECMO no Instituto da Criança Prof. Pedro de Alcantara do Hospital das Clínicas da Faculdade de Medicina da USP, discutindo seus aspectos clínicos e técnicos.

\section{Relato de caso}

Recém-nascido de ASP branco, masculino, 4.950g, nascido de parto vaginal com 39 semanas de idade gestacional, foi transferido no segundo dia de vida por insuficiência respiratória grave por Síndrome de Aspiração Meconial (SAM). Por ocasião da transferência, foi introduzido um dreno tubular sob selo d'água em hemitórax esquerdo para tratamento de um pneumotórax. O tratamento inicial consistiu em ventilação mecânica convencional com Fração Inspirada de Oxigênio $\left(\mathrm{FiO}_{2}\right)$ 1,0, freqüência respiratória (FR) 60, pressão expiratória final (Peep) $4 \mathrm{mmHg}$, pressão inspiratória (PINSP) $25 \mathrm{mmHg}$. A gasometria arterial sob esses parâmetros indicou pH 7,6; $\mathrm{PO}_{2} 45,4 ; \mathrm{PCO}_{2}$ 52,7; $\mathrm{SatO}_{2} 86 \%$. O ecocardiograma demonstrou sinais de Hipertensão Pulmonar grave. O ultra-som de crânio e os testes de coagulação sangüínea foram normais. Iniciou-se a administração de drogas inotrópicas (Dopamina e Dobutamina) em consequiência de hipotensão arterial e má perfusão periférica. Após 4 horas de tratamento, não houve melhora dos parâmetros respiratórios, sendo iniciada a administração de Óxido Nítrico inalatório (NOi), 20 p.p.m., aumentando-se a concentração de acordo com a necessidade até 30p.p.m. A gasometria arterial após 18 horas em NOi revelou pH 7,5; $\mathrm{PO}_{2} 125,9 ; \mathrm{PCO}_{2} 50,2 ; \mathrm{SatO}_{2} 98 \%$. Os parâmetros gasométricos pioraram progressivamente, apesar dos altos parâmetros ventilatórios e da concentração elevada de Noi. Como não observamos melhora da oxigenação apesar do máximo tratamento empregado, indicamos ECMO. A gasometria imediatamente anterior ao início da ECMO revelou $\mathrm{pH}$ 7,48; $\mathrm{PO}_{2} 43,9 ; \mathrm{PCO}_{2} 58,9$.

\section{Evolução da ECMO}

Realizamos a canulação da Artéria Carótida Direita e Veia Jugular Direita através de uma cervicotomia (Figura 1). Utilizamos uma cânula arterial $12 \mathrm{~F}$ e cânula venosa $14 \mathrm{~F}$. Foi iniciado o ECMO venoarterial, conforme a técnica descrita classicamente ${ }^{1,2}$. O circuito para ECMO, a bomba de rolete e o circulador de água utilizados foram fornecidos por Braile Biomédica. A membrana utilizada foi AVECOR 0800 .
O fluxo da circulação extracorpórea foi inicialmente mantido em $120 \mathrm{cc} / \mathrm{kg}$ e diminuído conforme a tolerado pela criança, com o objetivo de manter a $\mathrm{PO}_{2}$ entre 70 e 90 $\mathrm{mmHg}$ e a $\mathrm{PCO}_{2}$ entre 40 e $50 \mathrm{mmHg}$. A ventilação mecânica foi mantida com parâmetros mínimos, ou seja, $\mathrm{FiO}_{2}$ 0,3; FR 12/min; Peep 5mmHg; e Pinsp 20mmHG. O Tempo de Coagulação Ativado (TCA) foi mantido entre 180 e 220 segundos por meio da titulação de heparina. A contagem de plaquetas foi mantida acima de $100.000 / \mathrm{mm}^{3}$ e o hematócrito acima de $40 \%$, sendo realizadas transfusões quando necessárias.

No terceiro dia de curso de ECMO foi administrado surfactante exógeno de origem porcina, na dose de 100mg/ $\mathrm{kg}$, dose que foi repetida após 12 horas, segundo protocolo institucional de investigação do uso de surfactante na SAM. Seis horas após a administração da primeira dose do surfactante, observamos significativa melhora da complacência pulmonar, traduzida pelo clareamento de ambos os pulmões à radiografia, maior expansibilidade pulmonar à ventilação mecânica e rápida diminuição do fluxo de circulação extracorpórea (Figuras 2 e 3). O RN pôde ser decanulado no $5^{\circ}$ dia de ECMO. O tempo total do RN em ECMO foi de 116 horas.

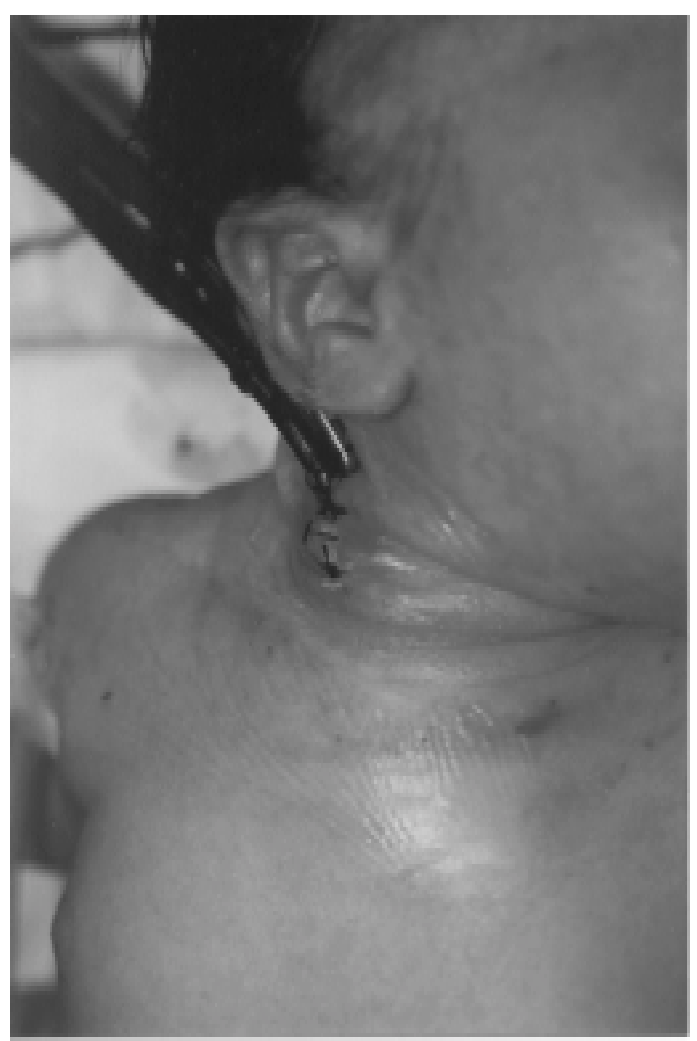

Figura 1 - Cânula arterial e venosa em incisão cervical direita para ECMO veno-arterial 


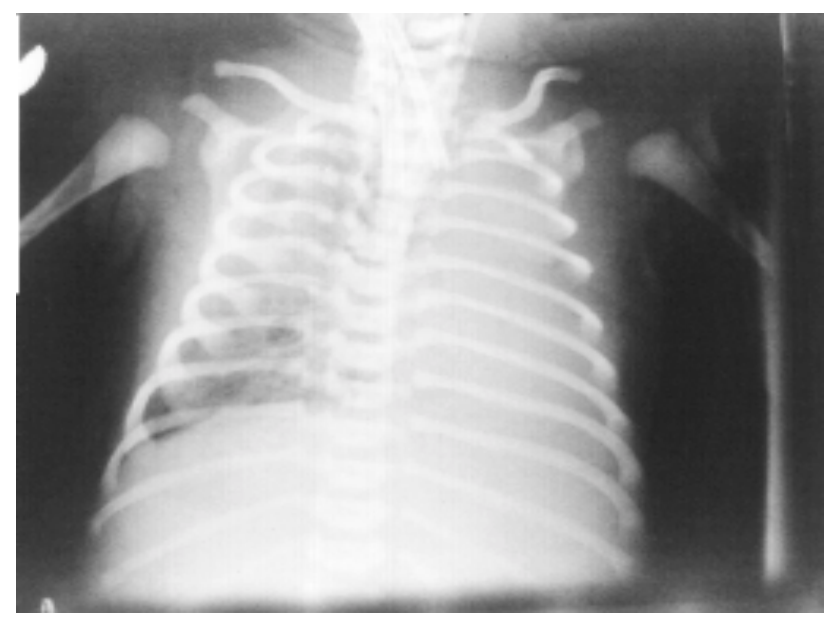

Figura 2 - Radiografia de tórax de recém-nascido no $4^{\circ}$ dia de ECMO, antes da administração de surfactante

\section{Complicações}

Não foram observadas complicações mecânicas. No segundo dia de ECMO observamos hemoglobinúria que clareou espontaneamente, diminuindo-se of fluxo de ECMO. Não houve complicações hemorrágicas. Um pequeno nódulo de $1,5 \mathrm{~cm}$ foi observado em adrenal direita em ultrasonografia de controle pós-decanulação, sugerindo uma hemorragia, mas sem repercussões clínicas. Durante a ECMO, Serratia marcescens foi isolada em hemocultura, tendo sido tratada com Cefalosporina com boa resposta. $\mathrm{O}$ $\mathrm{RN}$ foi extubado no $3^{\circ}$ dia pós-ECMO, mas necessitou reintubação devido a obstrução traqueal alta por laringite.

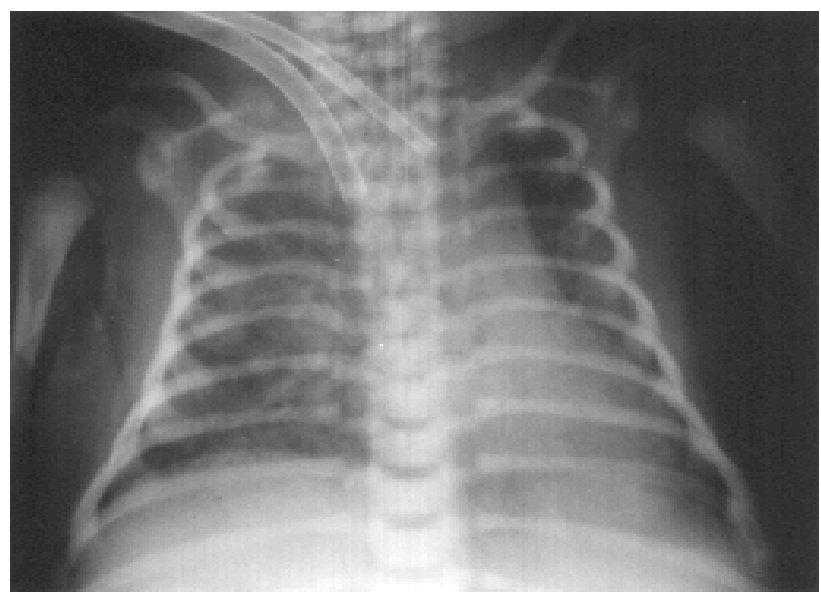

Figura 3 - Radiografia de tórax de recém-nascido no $4^{\circ}$ dia de ECMO, 6 horas após a administração de surfactante
O RN necessitou finalmente de uma traqueostomia pela gravidade dessa laringite, após o que permaneceu em ar ambiente. Recebeu alta sem traqueostomia.

\section{Discussão}

A ECMO é indicada no tratamento de RNs portadores de insuficiência respiratória grave que não respondem a outras terapias existentes, desde $1986^{1-3}$. É uma tecnologia complexa, trabalhosa, que requer um treinamento prévio adequado. Nas últimas duas décadas, o uso de ECMO cresceu de forma acentuada, tornando-se tratamento rotineiro em UTIs pediátricas terciárias, permitindo uma sobrevida de até $95 \%$ em crianças que teriam um índice mortalidade presumida superior a $80 \%{ }^{4}$.

Com o aprimoramento da técnica, o emprego de ECMO se tornou muito mais seguro e eficiente. Nas patologias respiratórias típicas do $\mathrm{RN}$, como raramente há necessidade de suporte cardiovascular por falência cardíaca, é possível realizar a técnica de ECMO veno-venosa. Essa técnica consiste na utilização de um catéter de duplo lúmen, que pode ser introduzido por punção ou dissecção, pela veia jugular interna até o átrio direito, para drenagem e reinfusão do sangue durante a circulação extracorpórea. Essa técnica mais simplificada evita a ligadura da artéria carótida e tem se mostrado igualmente eficiente e segura. Atualmente é a primeira opção técnica em muitos centros ${ }^{5}$.

Os benefícios do emprego de ECMO em casos selecionados não se limitam ao aumento da sobrevida, especialmente no período neonatal. Sabe-se que RNs tratados com ECMO têm menor incidência de seqüelas respiratórias e 
cardíacas, quando comparados a RNs de mesma gravidade tratados por métodos convencionais. Esses RNs também necessitam menos acompanhamento ambulatorial e por menor tempo, bem como um menor número de reinternações ${ }^{6}$.

As complicações de ECMO podem ser divididas em dois tipos: mecânicas, isto é, aquelas que ocorrem no próprio circuito extracorpóreo, e do paciente. Quanto maior o tempo em ECMO, maior a incidência de complicações.

A incidência, gravidade e as consequiências das complicações mecânicas são diretamente relacionadas à experiência acumulada pela equipe de ECMO. Em centros de referência em ECMO, essas complicações são infreqüentes e geralmente não têm repercussão no resultado final ${ }^{7}$.

As complicações mecânicas como coágulos no circuito, embolia gasosa, rachadura em componentes do circuito e falência do oxigenador são mais freqüentes durante ECMO em crianças maiores e adultos do que durante a ECMO neonatal.

A maioria das complicações clínicas são conseqüentes a três alterações que necessariamente ocorrem durante a ECMO: a anticoalgulação, as interações do sangue com as superfícies artificiais do circuito e as mudanças no padrão de fluxo sangüíneo. As complicações clínicas mais comuns em ECMO neonatal, de acordo com os dados cumulativos da ELSO (Extracorporeal Life Support Organization), são retenção hídrica com necessidade de hemofiltração, convulsões, hemorragia intracraniana, hemólise, hipertensão arterial, infecção e elevação de creatinina sérica.

Com exceção da hemorragia ou infarto intracraniano, a maior parte das complicações são controláveis com relativa facilidade e, geralmente, não alteram significativamente $o$ resultado final. As hemorragias são as principais complicações do suporte extracorpóreo prolongado, sendo a hemorragia intracraniana (HIC), principalmente em recém-nascidos, a complicação mais grave.

\section{Critérios para indicação de ECMO}

Os critérios para indicação de ECMO no nosso grupo são os mesmos utilizados pela maioria dos centros de ECMO, ou seja:

a) critérios genéricos: $\mathrm{RN}$ maior que $2.500 \mathrm{~g}$ ou acima de 35 semanas de idade gestacional, sem coagulopatia, sem cardiopatia congênita, em ventilação mecânica por menos de 15 dias, sem anomalias cromossômicas ou congênitas incompatíveis com a vida.

b) critérios específicos: índice de oxigenação $\geq 40$, deterioração aguda do quadro clínico, parada ou falência cardíaca, barotrauma, gradiente alvéolo-arterial de oxigênio $\geq 620 \mathrm{mmHg}$ e ausência de resposta ao todos os outros tratamentos disponíveis.

Este último critério foi o mais decisivo para indicação de ECMO neste caso.
Na tentativa de abreviar o duração do suporte extracorpóreo, foi utilizado o surfactante porcino. O emprego de surfactante para esse fim tem sido recomendado por alguns autores, com a finalidade de reduzir o tempo de evolução em $\mathrm{ECMO}^{8-12}$.

Stillerman et al. estudaram o efeito da administração de surfactante bovino em RNs, logo após o início da ECMO, demonstrando uma redução significativa na duração do suporte extracorpóreo ${ }^{11}$.

Em um estudo randomizado e controlado feito por Lotze e cols., foi observado que a utilização de múltiplas doses de surfactante bovino levaram a uma melhora significativa na função pulmonar de RNs a termo submetidos a ECMO. Os autores verificaram ainda melhora na complacência pulmonar, aumento na concentração de proteína de surfactante A no aspirado traqueal e uma diminuição da necessidade do suporte extracorpóreo ${ }^{9}$.

O melhor momento para a administração de surfactante não está bem definido. Optamos por administrar a primeira dose apenas no $4^{\circ}$ dia de ECMO, quando a criança se encontrava sem edema periférico, e a radiografia de pulmão revelava o início da re-aeração pulmonar. De fato, seis horas após a administração de surfactante, observamos sensível melhora na função pulmonar, permitindo rápida diminuição do suporte extracorpóreo (Figura 4).

Com relação às complicações do uso de surfacatante, conforme os relatos da literatura, não constatamos efeitos adversos que pudessem interferir com a evolução da criança.

A principal resistência quanto à criação e à manutenção de um programa de ECMO nos hospitais pediátricos brasileiros é relacionada ao custo do procedimento. Tal fato é conseqüente da divulgação de orçamentos superestimados e não condizentes com a realidade do Brasil, por institui-

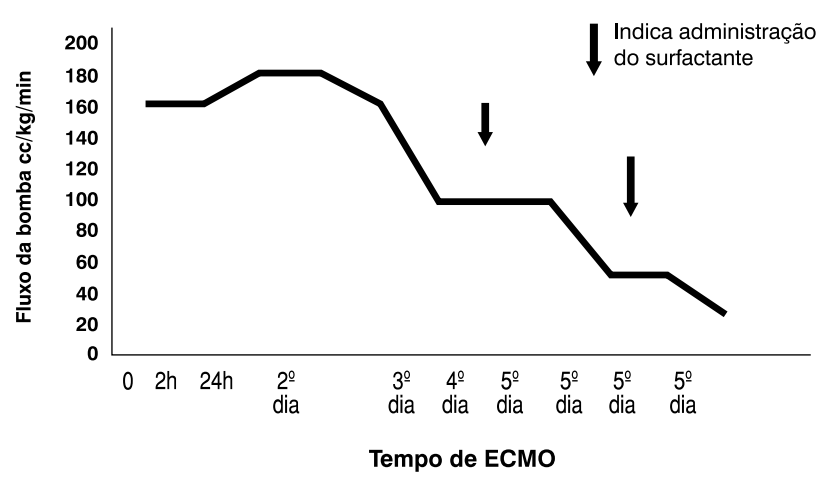

Figura 4 - Fluxo de ECMO durante os 5 dias; observar a diminuição do fluxo após a administração de surfactante 
ções estrangeiras, revistas e livros especializados. Tais orçamentos acabam gerando a idéia de um procedimento de custo proibitivo e impraticável. As Tabelas 1 e 2 fazem uma comparação, em dólares americanos, dos custos habitualmente descritos nessas publicações e os da nossa instituição ${ }^{13}$.

Tabela 1 - Comparação dos custos de ECMO nos EUA e Brasil. (Material permanente)

\begin{tabular}{lrr}
\hline Equipamento & Custo EUA U\$ & Custo ICr U\$ \\
\hline 1. Bomba & $10.000,00$ & $3.200,00$ \\
2. Sensor de pressão & $1.000,00$ & 150,00 \\
3. Bladder box & $3.000,00$ & Adaptado \\
4. Circulador de água & $3.000,00$ & $1.900,00$ \\
5. Console com bateria & $2.500,00$ & $1.500,00$ \\
6. Máquina de TCA & $3.000,00$ & $3.000,00$ \\
Total & $\mathbf{2 2 . 5 0 0 , 0 0}$ & $\mathbf{9 . 7 5 0 , 0 0}$ \\
\hline
\end{tabular}

Um dos maiores desafios na implantação de um novo programa de ECMO é treinar uma equipe de "Especialistas em ECMO", profissional-chave para o sucesso do programa. Esse profissional é responsável pelos cuidados contínuos com o circuito e com os controles de heparinização e trocas gasosas pela membrana. Em centros de ECMO, enfermeiros e perfusionistas podem se qualificar para a tarefa após treinamento adequado. No nosso caso, optamos por inicialmente estabelecer a equipe apenas com perfusionistas, pois estes são mais familiarizados com a técnica e possuem maior conhecimento da fisiologia da Circulação Extracorpórea. Entretanto, os perfusionistas implicam em

Tabela 2 - Comparação de custos, material descartável (avaliação para 20 casos/ano)

\begin{tabular}{lrr}
\hline Equipamento & EUA (U\$) & ICr (U\$) \\
\hline 1. Oxigenadores (20casos/ano) & $11.600,00$ & $11.600,00$ \\
2. Circuitos sob medida 20 casos/ano & $10.000,00$ & $1.000,00$ \\
3. Catéteres /ano & $12.000,00$ & $12.000,00$ \\
4. Tubos de TCA/ano & $5.000,00$ & $5.000,00$ \\
Total: & $38.600,00$ & $29.600,00$ \\
\hline Total: Permanente + Descartáveis & $61.000,00$ & $39.350,00$ \\
\hline
\end{tabular}

Obs.: fonte dos custos nos EUA: Bartlett RH, et al. Economics of Extracorporeal Life Support. In: Zwischenberger JB, Bartlett RH. ECMO: Extracorporeal Cardiopulmonary Support in Critical Care. Michigan: Extracorporeal Life Support Organization;1995. p.533-50. um custo adicional para o programa, pois são profissionais especializados que habitualmente não estão à disposição de hospitais pediátricos que não realizam cirurgias cardíacas. Apesar disso, em instituições com características semelhantes às nossas, recomendamos que tal orientação seja seguida ${ }^{14}$.

\section{Agradecimentos}

Os autores agradecem a dedicação e o competente trabalho do grupo de perfusionistas de ECMO: Flavia Cristina Gomes Alves, Maria Regina Gonçalves de Barros, Ana Paula Noronha da Silva, Nelma Israelina Teodoro, Alessandra Cristina Girasol, Adriana de Jesus Savacini e Ana Paula Freitas.

\section{Referências bibliográficas}

1. Bartlett RHL, Andrews AF, Toomasian JM. Extracorporeal membrane oxygenation for newborn respiratory failure: 45 cases. Surgery 1982;92:425-31.

2. Fauza DO, Maksoud-Filho JG. ECMO (Extracorporeal Membrane Oxygenation). In: Maksoud JG. Cirurgia Pediátrica. 1st ed. Rio de Janeiro: Revinter; 1998. p. 163-85.

3. Maksoud-Filho JG. ECMO (Circulação Extracorpórea por Membrana). Pediatr Moderna 2000;36:68-76.

4. Kanto WP Jr, Bunyapen C. Extracorporeal membrane oxygenation. Controversies in selection of patients and management. Clin Perinatol 1998;25:123-35.

5. Anderson HL, Snedecor SM, Otsu T, Bartlett RH. Multicenter comparison of conventional venoarterial access versus venovenous double lumen catheter access in newborn infants undergoing extracorporeal membrane oxygenation. J Pediatr Surg 1993;28:530-4

6. Pearson GD, Short BL. An economic analysis of extracorporeal membrane oxygenation. J Intens Care Med 1986;2:116-9.

7. Zwischemberger JB, Nguyen TT, Upp Jr JR, Bush PE, Cox CS $\mathrm{Jr}$, De Losh T, Broemly L. Complications of neonatal extracorporeal membrane oxygenation. Colective experience from Extracorporeal Life Support Organisation. J Thorac Cardiovasc Surg 1994;107:838-48.

8. Short BL. Clinical management of the nonatal ECMO patient. In: Arensman RN, Cornish JD. Extracorporeal life support. 1st ed. Boston: Blackwell Scientific Publications; 1993. p.195-206.

9. Lotze A, Knight GR, Martin GR. Improved pulmonary outcome after exogenous surfactant therapy for respiratory failure in term infants requiring extracorporeal membrane oxygenation. J Pediatr 1993;122:261-8.

10. Lotze A, Mitchell BR, Bulas DI, Zola EM, Shalwitz RA, Gunkel JH. Multicenter study of surfactant (beractant) use in the treatment of term infants with severe respiratory failure. Survanta in Term Infants Study Group. J Pediatr 1998;132:40-7.

11. Stillerman LR, Gunn SB, Hart JC, Engle WA. Effects of exogenous surfactant on neonates supported by extracorporeal membrane oxygenation. J Perinatol 1997;17:262-5.

12. Diniz EMA, Costa MTZ. Outras indicações de surfactante exógeno. Pediatr Moderna 2000;36:25-9. 
13. Bartlett RH, Schumacher RE, Chapman RA. Economics of extracorporeal life support. In: Zwichemberger JP, Bartlet RH. ECMO: Extracorporeal cardiopulmonary support in critical care. Michigan: Extracorporeal Life Support Organization; 1995, p.533-50.

14. Wetmore NE, Bartlett RH, Gazzaniga AB. Extracorporeal membrane oxygenation (ECMO): a team aproach in critical care and life support research. Heart Lung 1979;8:288-93.
Endereço para correspondência:

Dr. João Gilberto Maksoud Filho.

R. Jericó, 59, ap. 11 - CEP 05435-040 - São Paulo, SP

Fone: (11) 815.4627 - Fax: (11) 3069.8668

E-mail:maksoudf@icr.hcnet.usp.br 